\title{
1 A dual PCR-based sequencing approach for the identification and \\ 2 discrimination of Echinococcus and Taenia taxa
}

3

4 Ghalia Boubaker ${ }^{\mathrm{a}, \mathrm{b}} 1$, Irina Marinova ${ }^{\mathrm{a}, \mathrm{c}} 1$, Francesca Gori ${ }^{\mathrm{d}}$, Amani Hizemª, Norbert Müller ${ }^{\mathrm{a}}$,

5 Adriano Casulli ${ }^{\mathrm{e}}$, Luis Enrique Jerez Puebla ${ }^{\mathrm{f}}$, Hamouda Babba $^{\mathrm{b}}$, Bruno Gottstein ${ }^{\mathrm{a}^{*}}$, Markus

$6 \quad$ Spiliotis $^{\mathrm{a}}$

$7 \quad$ a Institute of Parasitology, University of Bern, Bern, Switzerland

$8{ }^{\mathrm{b}}$ University of Monastir, Faculty of Pharmacy, Department of Clinical Biology B, Laboratory

9 of Medical and Molecular Parasitology-Mycology (LR12ESO8), Monastir, Tunisia

$10{ }^{\mathrm{c}}$ Department of Parasitology and Tropical Medicine, National Center of Infectious and

11 Parasitic Diseases, Sofia, Bulgaria

$12{ }^{\mathrm{d}}$ Institute of Parasitology, University of Zurich, Zurich, Switzerland

13 e Department of Infectious, Parasitic and Immunomediated Diseases, Istituto Superiore di

14 Sanità, viale Regina Elena 299, 00161 Rome, Italy

$15{ }^{\mathrm{f}}$ Pedro Kouri Institute, Autopista Novia del Mediodia, La Lisa, La Habana, Cuba

16

$17{ }^{1}$ These authors equally contributed to the performance of the study

18

19

*Corresponding author: Professor Bruno Gottstein, Institute of Parasitology, University of

Berne, Länggassstrasse 122, CH-3012 Bern, Switzerland.

21

*E-mail: bruno.gottstein@vetsuisse.unibe.ch 


\section{ABSTRACT}

Reliable and rapid molecular tools for the genetic identification and differentiation of Echinococcus species and/or genotypes are crucial for studying spatial and temporal transmission dynamics. Here, we describe a novel dual PCR targeting regions in the small $(r r n \mathrm{~S})$ and large ( $r r n \mathrm{~L})$ subunits of mitochondrial ribosomal RNA (rRNA) genes, which enables (i) the specific identification of species and genotypes of Echinococcus ( $r r n \mathrm{~S}+\mathrm{L}-$ PCR) and/or (ii) the identification of a range of taeniid cestodes, including different species of Echinococcus, Taenia and some others (17 species of diphyllidean helminths). This dual PCR approach was highly sensitive, with an analytical detection limit of 1 pg for genomic DNA of Echinococcus. Using concatenated sequence data derived from the two gene markers (1225 bp), we identified five unique and geographically informative single nucleotide polymorphisms (SNPs) that allowed genotypes (G1 and G3) of E. granulosus sensu stricto to be distinguished, and 25 SNPs that allowed differentiation within E. canadensis (G6/7/8/10). In conclusion, we propose that this dual PCR-based sequencing approach can be used for molecular epidemiological studies of Echinococcus and other taeniid cestodes.

Keywords: Echinococcus spp.; Taenia spp., PCR; specific and genotypic identification; 12S and $16 \mathrm{~S}$ nuclear ribosomal RNA genes. 


\section{Introduction}

Echinococcosis is a parasitic disease caused by the infection with tiny tapeworms of the genus Echinococcus, with E. granulosus and E. multilocularis being the species of principal medical importance. The life cycles of Echinococcus species predominantly involve a definitive host and an intermediate host, each harbouring different developmental stages of the parasite. The larval stage (metacestode) develops mainly within the liver, but also in other organs, of the intermediate host, while adult worms parasitize mostly the small intestine of canids, and produce eggs, which are infective to intermediate hosts. Humans are accidental intermediate hosts [1]. The two main and pathologically distinct forms of disease in humans are alveolar echinococcosis (AE) caused by E. multilocularis, and cystic echinococcosis (CE) caused by E. granulosus sensu lato (s.1.) [1].

Formerly, a high degree of genetic diversity was found within E. granulosus s.1.; thus, 10 genotypes (G1-G10) have been documented based on analyses of mitochondrial DNA (mtDNA) regions [2-4]. Subsequently, the taxonomy of the genus Echinococcus was reviewed to clarify the systematic status of various species and genotypes [5,6]. This revision has led to the attribution of species status to some of the E. granulosus genotypes. Thus, $E$. granulosus is now recognized as a complex of four cryptic species: E. granulosus sensu stricto (s.s.) (genotypes G1/2/3), E. equinus (G4), E. ortleppi (G5) and E. canadensis (G6/7/8/10) [5,6]. A large number of comprehensive analyses of mitochondrial [6,7] and nuclear genome targets [8,9] in Echinococcus has not assisted in fully resolving some nomenclature and taxonomic issues. For instance, there is still controversy as to whether $E$. canadensis (G6/7/8/10) comprises at least three species [9-15]. In contrast to E. granulosus s.l., the genetic differences among E. multilocularis isolates from different geographical areas are minimal $[16,17]$.

The molecular epidemiology of the genus Echinococcus is of substantial importance, since it allows a better understanding of the spatio-temporal distribution patterns of individual species/genotypes, and their relative contribution to human alveolar and cystic echinococcosis. Such investigations also aid in the planning and implementation of disease control measures [18-20]. Indeed, cumulative 'genotyping' data for human isolates have revealed that E. granulosus s.s. $(\mathrm{G} 1 / 2 / 3)$ and E. canadensis $(\mathrm{G} 6 / 7)$ are the two commonest species responsible for human CE [19,21]. (1) 
To date, molecular phylogenetic analyses have relied on DNA sequence data obtained from partial single loci (ranging from 250 to $500 \mathrm{bp}$ in length), particularly from mitochondrial DNA loci, such as cytochrome $c$ oxidase subunit $1(\operatorname{cox} 1)[17,22,23], \mathrm{NADH}$ dehydrogenase subunit 1 (nad1) [22-25], ATP synthase subunit 6 (atp6) [26] and the small subunit $(S S U)$ of the rRNA gene [27,28]. However, these relatively short sequences have insufficient discriminatory power to resolve fine scale taxonomic peculiarities (e.g. placement of strains/haplotypes into clades) within the E. granulosus complex, particularly in relation to E. granulosus s.s. $(\mathrm{G} 1 / 2 / 3)$ and E. canadensis $(\mathrm{G} 6 / 7 / 8 / 10)$. Thus, the selection of gene markers useful that allow the accurate identification and differentiation of species and genotypes (intraspecific variants) has been a major challenge for systematic and molecular epidemiological studies of Echinococcus_and other taeniids.

Recently, we developed a multiplex PCR (Egc-mPCR) as a rapid and reliable test for the identification of all recognized species within the E. granulosus complex [29]. However, like other one-step techniques, such as high-resolution melting (HRM) [30], loop-mediated isothermal amplification (LAMP) [31] and multiplex PCR [32], the Egc-mPCR did not permit further extensive and deeper systematic analyses.

In the present study, we developed a dual PCR-coupled sequencing methods (using rrnS and $r r n \mathrm{~L}$ ) for the identification and characterization of all species and genotypes of Echinococcus. To this end, we designed two primer sets: (i) the primer pair $r r n S-F$ and $\mathrm{R}$ is highly specific for the genus Echinococcus and (ii) the $r r n \mathrm{~L}-\mathrm{F}$ and $r r n \mathrm{~L}-\mathrm{R}$ amplifies a region within $r r n \mathrm{~L}$ from taeniid cestodes, including all recognized species and genotypes of Echinococcus. We assessed and validated the analytical sensitivity and specificity of the dual PCR using a panel of Echinococcus reference DNAs (representing cysts or eggs) and DNA samples from other helminths (cestode, nematode and trematode species). 


\section{Materials and methods}

\subsection{Ethical aspects}

Parasite specimens of animal origin were derived from an existing bio-bank collection of

the Institute of Parasitology (Bern, Switzerland), and some of these samples had already been used in previous studies $[29,33]$. Samples were collected within the framework of governmentally regulated meat inspections in slaughterhouses; respective specimens were sent to the laboratory of Parasitology for parasitological and molecular identification. Parasite samples from human patients were derived from an existing collection of Echinococcus DNA-biobank at the EPS Fattouma Bourguiba Hospital in Monastir/Tunisia. This collection had been designed and ethically approved for basic research studies.

\subsection{Biological materials}

A panel of Echinococcus genomic DNA (gDNA) samples was used to assess the sensitivity and specificity of the two PCR assays established here. These DNAs had been individually extracted from metacestodes (hydatid cysts for E. granulosus, metacestode tissue for E. multilocularis or polycystic tissue for E. vogeli). Table 1 provides detailed information (including geographic origin, intermediate host and metacestode localization) about these Echinococcus reference gDNAs. The genetic identity of the samples (species/genotypes) representing the E. granulosus complex was determined based on the amplification profile of our previously published multiplex PCR [29], whereas the E. multilocularis and E. vogeli samples had been identified previously via coxl and nadl sequencing [34]. Additional taeniid and/or E. multilocularis egg gDNA samples were obtained from the institutional DNAcollection of the Institute of Parasitology, University of Zürich (Switzerland). Taeniid eggs (including E. multilocularis) were isolated using a sieving-flotation technique [35], and species identification was performed using the triplex-PCR published by Trachsel et al. [36]. Upon isolation/purification of eggs, 8 faecal samples yielded only E. multilocularis (3 dogs and 5 foxes), 2 samples only E. granulosus s.l. (1 dog and 1 wolf), and 5 faecal samples (2 wolves and 3 fox) only Taenia species.

A panel of gDNA samples isolated from other helminths included 1 trematode species (Dicrocoelium dendriticum), 7 cestode species (Mesocestoides corti, Taenia hydatigena, $T$. crassiceps, T. polyacantha, T. multiceps, T. taeniaeformis and Hymenolepis diminuta) and 3 
nematode species (Ascaris suum, Toxocara canis and Ancylostoma duodenale). These samples were used to test for potential cross-amplification in the dual PCR established here. We used 50 ng of each DNA per PCR.

\subsection{Primer design and PCR conditions}

All available complete mitochondrial genome sequences for Echinococcus (species/genotypes) and the entire mitochondrial genomes for other helminths (13 cestodes, 4 nematodes and 2 trematodes) of human or dog origin were retrieved from GenBank (National Centre for Biotechnology Information, NCBI), imported and aligned using ClustalW in the program BioEdit (version 7.0.9). Complete data on accession numbers and the species names are given in Supplementary Table A.1. Two primer pairs were designed: $r r n S-F / r r n S-R$ for all Echinococcus species and genotypes, and $r r n \mathrm{~L}-\mathrm{F} / r r n \mathrm{~L}-\mathrm{R}$ for all Echinococcus species members and 13 taeniid cestodes (corresponding to the selection that was included in the nucleotide alignment). The sequences and characteristics of the primers are shown in Table 2.

The QIAGEN Multiplex PCR Kit that contains HotStarTaq DNA Polymerase (Qiagen) was used for the amplification of partial sequences of the $r r n S$ and $r r n L$. PCR reaction mix $(50 \mu \mathrm{l})$ consisted of $25 \mu \mathrm{l}$ of multiplex PCR master mix, $0.5 \mu \mathrm{M}$ of each primer, and $19 \mu \mathrm{l}$ $\mathrm{H}_{2} \mathrm{O}$ and $5 \mu$ l of template DNA. Cycling conditions were: $95^{\circ} \mathrm{C} / 15 \mathrm{~min}, 40$ cycles $\left(94^{\circ} \mathrm{C} / 30 \mathrm{~s}\right.$, $56^{\circ} \mathrm{C}(r r n S)$ or $\left.58^{\circ} \mathrm{C}(r r n L) / 30 \mathrm{~s}, 72^{\circ} \mathrm{C} / 30 \mathrm{~s}\right)$ and $72^{\circ} \mathrm{C} / 10 \mathrm{~min}$. The PCR products were detected by electrophoresis in $1.5 \%$ agarose gels stained with ethidium bromide and purified with the High Pure PCR Product Purification kit (Roche Applied Science) according to the manufacturer's instructions. Subsequently, they were sequenced using an automated DNA sequencer (Applied Biosystems, ABI 3130× I Genetic Analyzer Sequencer) using the same primers as use for amplification. Resultant sequences were edited using BioEdit (version 7.0.9.0). The Basic Local Alignment Search Tool (BLAST) of NCBI was used to confirm the identity of each sequence.

To verify nucleotide variations between species/genotypes, we repeated the PCR-based sequencing for a representative set of samples, but employing proofreading polymerase ( $P f u$ DNA Polymerase; Promega). Here, PCRs were performed in $40 \mu 1$ reaction mixes containing $0.5 \mu \mathrm{M}$ each forward and reverse primers, $100 \mu \mathrm{M}$ dNTPs mix $(10 \mathrm{mM}), 0.05$ units $\mu \mathrm{l}-1$ Pfu DNA polymerase in $1 \times P f u$ DNA polymerase Buffer and $2 \mu 1$ of DNA. Cycling conditions were: initial denaturation $95^{\circ} \mathrm{C} / 2 \mathrm{~min}, 30$ cycles of $95^{\circ} \mathrm{C} / 30 \mathrm{~s}, 56^{\circ} \mathrm{C}(\mathrm{rrnS})$ or $58^{\circ} \mathrm{C}(\mathrm{rrnL}) / 30$ s and $72^{\circ} \mathrm{C} / 30 \mathrm{~s}$ ) followed by a final extension step of $72^{\circ} \mathrm{C} / 5 \mathrm{~min}$. 


\subsection{Analytical sensitivity of the assay}

To determine the analytical sensitivity of the dual PCR, different amounts of E. granulosus s.s. (G1) DNA (5 ng, 1 ng, 100 pg, 10 pg, 1 pg, $100 \mathrm{fg}, 10 \mathrm{fg}$ and $1 \mathrm{fg}$ ) was tested in the PCRs described in subsection 2.3.

\subsection{Phylogenetic analysis and tree construction}

All partial $r r n S$ and $r r n L$ sequences obtained from amplicons for Echinococcus reference gDNA samples (see Table 1) were edited and aligned using BioEdit software (version 7.0.9.0.). The sequences were also mapped to reference mitochondrial genomes representing Echinococcus (all accession numbers given in Supplementary Table 1). Phylogenetic analyses were performed on individual partial gene sequences as well as on the concatenated $(r r n S+$ $r r n L$ ) data set employing MEGA software (Molecular Evolutionary Genetics Analysis 3.1; http://www.megasoftware.net). To reconstruct phylogenetic trees, we used tree-building methods: neighbour joining (NJ) [37] [38], maximum parsimony (MP) [39-43], and maximum likelihood (ML) $[38,41,44]$. To evaluate the robustness of each tree, a Bootstrap (BS) values were determined by bootstrap resampling (BS) [45] (1000 random repetitions (NJ), 100 replications (MP and ML), and 100\% and 70\% were considered as high and moderate $\mathrm{BS}$ values.

\section{Results}

\subsection{Sensitivity and specificity of PCRs}

The analytical sensitivity of the two PCRs was assessed by using various concentrations of E. granulosus s.s. (G1) DNA template. As shown in Figure 1, the sensitivity analysis revealed a similar detection limit of $1 \mathrm{pg}$ of gDNA template for both primer pairs (Fig. 1). The specificity of the PCRs was assessed using 11 gDNA control samples derived from trematodes, nematodes and non-Echinococcus cestodes. Results showed complete agreement between predicted and observed specificity of the $r r n S-P C R$. Indeed, the $r r n S-F / r r n S-R$ primer pair did not amplify from DNA from heterologous helminth species tested $(n=11)$, indicating the specificity of these primers for the specific amplification from DNA of Echinococcus species and genotypes. As shown in Fig. 2, the primer pair $r r n \mathrm{~L}-\mathrm{F} / r r n \mathrm{~L}-\mathrm{R}$ is 
cestode-specific, amplifying only the target sequences (648 bp) from DNA from all 7 cestode species tested here. The dual PCR did not show any cross-amplification from any of the DNA samples from nematodes or trematodes.

\subsection{Sequencing of amplicons for Echinococcus genotyping}

Both $r r n S$ and $r r n \mathrm{~L}$ gene fragments of expected lengths were amplified from all Echinococcus reference DNA samples (Table 1; Fig. 3). Sequence analyses of these fragments allowed the identification of and differentiation among all Echinococcus species and genotypes, including E. granulosus s.s. (G1) and E. granulosus s.s. (G3) and members of the E. canadensis (G6-G10) clade. Three (positions 152, transition A/G; position 183, transition G/A and position 533, transversion T/G) and two (position 338, transition C/T; position 580 , transition $\mathrm{A} / \mathrm{G}$ ) stable and unique discriminatory single nucleotide polymorphisms (SNPs) were detected in the $r r n \mathrm{~S}$ and $r r n \mathrm{~L}$ regions, respectively. This subset of 5 unique SNPs allowed the accurate distinction between E. granulosus s.s. (G1) and E. granulosus s.s. (G3) from distinct geographical areas (Tunisia, Algeria, Italy and Greece). Four out of seven $E$. granulosus s.s. $(\mathrm{G} 1 / 2 / 3)$ isolates were identified as $E$. granulosus s.s. (G3) (Table 1). For differentiation within E. canadensis (G6/7/8/10), we found 25 SNPs; 12 are present in $r r n S$ and 13 in the $r r n \mathrm{~L}$ markers (Table 3). Phylogenetic trees constructed based on the partial $r r n S, r r n L$ and on concatenated $(r r n L+r r n S)$ partial sequences are displayed in Fig 4. Three isolates ( 2 from Tunisia and 1 from Algeria) clustered within E. granulosus s.s (G1), and four sequences (1 Tunisian, 1 Algerian, 1 Italian and 1 Greek) grouped within E. granulosus s.s (G3) with high bootstrap support of $100 \%$ (NJ/MP/ML)

Genomic DNA samples that were extracted from taeniid eggs were tested using the dual PCR assay. All samples were positive in the $r r n$ L-PCR and yielded a band of the expected size (648 bp), indicating the presence of taeniid cestode DNA in these samples. Sequencing revealed E. multilocularis in 8 samples (5 from foxes and 3 from dogs); E. canadensis (G7) was found in $1 \mathrm{dog}$, E. granulosus s.s. (G1) in 1 wolf, T. taeniaeformis DNA in 1 fox, $T$. hydatigena in 2 foxes and T. multiceps in 2 wolves. Using the rrnS-PCR, only samples containing E. multilocularis or E. granulosus s.l. DNA were test positive. For these samples, DNA sequencing results was in complete agreement with those obtained by sequencing the $r r n \mathrm{~L}$ gene region. 


\section{Discussion}

Several molecular tools have been developed for the genetic characterization of different species from the genus Echinococcus. The aim of the present study was to enhance the utility of a diagnostic PCR developed previously [29] to enable inter- and intra-species discrimination among all important members of the genus Echinococcus, and the detection of other non-Echinococcus cestodes. Although the previously described PCR [29] was inexpensive and easy to perform, the following limitations became apparent: it could not distinguish between E. granulosus s.s. genotypes (G1/3); it worked reliably only on DNA extracted from cysts; it was not sensitive enough to be used for the specific identification of eggs when these were present in low numbers [29]; extensive phylogenetic analysis was not possible because of limited informative nucleotide sites; and, finally, taeniids other than Echinococcus could not be detected. Similar issues might hamper other one-step or single species-specific detection systems [30-32].

Since it was shown that mitochondrial gene markers (sensitivities varying between $73.7 \%$ and $100 \%$ ) provide a better performance for the detection and identification of Echinococcus species than nuclear targets (sensitivity of 52.6\%) [46], we focused here on the identification of mitochondrial genome-derived markers. Furthermore, it is generally well-accepted that mitochondrial DNA evolves faster than the nuclear genome and appeared to render mitochondrial DNA more appropriate for genetic analyses of the genus Echinococcus [22,23,25-28]. In this study, we established a dual PCR assay for the specific detection of taeniids as well as Echinococcus. The rrnS-PCR detected all species and genotypes of Echinococcus included here, and the rrnL-PCR theoretically detected 17 cestode species (only 7 species were experimentally tested), including Echinococcus.

For the analysis of specificity, we did not test all helminth species that were included in the alignment of the mitochondrial genome sequences (32 species). However, based on aligned mitochondrial DNA sequence data, (i) the $r r n S-F / r r n S-R$ primer pair should also detect $E$. shiquicus, E. oligarthrus and E. felidis, and (ii) the $r r n \mathrm{~L}-\mathrm{F} / r r n \mathrm{~L}-\mathrm{R}$ primer pair detected other cyclophyllidean species included here (Mesocestoides corti, Taenia hydatigena, T. crassiceps, T. polyacantha, T. multiceps, T. taeniaeformis and Hymenolepis diminuta), and are thus proposed to amplify from other cestodes (T. serialis, T. solium, T. ovais, T. madoquae, $T$. regis, $T$. twitchelli, T. arctos, T. martis, Dipylidium caninum and Versteria mustelae). Since large differences in the primer binding sites are present between cestodes, on one hand, and trematodes or nematodes, on the other, no cross-amplification is expected. However, further experiments need to be performed to test these assumptions. 
The two PCRs were highly sensitive and showed a very low detection-limit in the range of 1pg Echinococcus DNA. This result is comparable to previously described PCR-based approaches for molecular diagnosis of E. granulosus s.1. [47,48] and/or E. multilocularis $[28,36]$ in the definitive hosts, where the presence of one egg was sufficient to give a specific signal (one egg contains approximately 8 pg of DNA [49]).

The PCRs allowed the discrimination between all Echinococcus species and genotypes included in this study, and phylogenetic trees constructed using either $r r n \mathrm{~S}, r r n \mathrm{~L}$ or concatenated sequence data produced similar topologies, in particular the close relationships between E. granulosus s.s. or E. canadensis genotype members [5]. Since the discrimination between G1 and G3 has been a controversial issue and has largely relied on the use of mitochondrial gene markers [26], many studies have allowed only E. granulosus s.s. to be defined, without a discrimination among genotypes G1, G2 and G3 [50,51]. Consequently, the real contribution of E. granulosus s.s. (G3) to human CE might be underestimated. On the other hand, one may raise the question as to how far discriminating among these conventional genotypes (i.e. G1-G3) makes sense in the context of large haplotype diversity. Future studies should help to better address these questions. The complete mitochondrial genome of $E$. granulosus s.s. (G3) was sequenced only a year ago [52] and was used in our study for primer design. Consequently, the PCRs established here should improve the genotyping by clarifying the specific and genotypic identity of isolates.

In most cases, molecular studies of Echinococcus from definitive and/or intermediate hosts have been carried out using different molecular tests, depending on the origin of the biological matrix used for analysis. However, it was recently shown that the genetic diversity of $E$. granulosus s.s. in intermediate animal hosts is greater than in definitive hosts [53]. This highlights the importance of including samples derived from both hosts in a given area to elucidate the population structures and the genetic complexity of Echinococcus, and to use an appropriate methodology. In our experiments, besides the high performance of the present PCRs on DNA extracted from Echinococcus larval stage, we also obtained successful and specific amplification of the partial $r r n S$ and $r r n L$ sequences from the 16 Echinococcus DNA samples derived from eggs. However, despite this promising potential of these PCRs to be applied for comparative assessments of the genetic variability of Echinococcus in intermediate and definitive hosts, future research should be conducted using a larger and more diverse panel of samples. The PCRs should also be tested on DNA isolated directly from faeces from Echinococcus-infected definitive hosts [54], or even from environmental samples, such as soil or water [55]. In this regard, the sensitivity of the present PCRs might be 
302 challenged, possibly requiring an enrichment of parasite DNA prior to performing molecular 303 analysis, as indicated recently by other workers [54].

304 In conclusion, we have established two effective PCRs, targeting regions of the 305 mitochondrial $r r n S$ and $r r n \mathrm{~L}$ genes. These tests can be applied for a high-resolution 306 characterization of all Echinococcus species and genotypes. In addition, the rrnL-PCR can be 307 used for the detection and identification of at least 17 cestode species other than 308 Echinococcus. 
310 Many thanks go to Professor Andrew Hemphill (Institute of Parasitology, University of 311 Bern, Switzerland) for critical reading of the manuscript. We thank Professor Peter Deplazes 312 (Institute of Parasitology, University of Zürich, Switzerland) for kindly providing reference 313 taeniid egg-DNA samples. The work has been supported by the Swiss National Science 314 Foundation (research grant no.31003A_160108/1) and Sciex project no. BGR_13.117. 


\section{References}

[1] J. Eckert, P. Deplazes, Biological, Epidemiological, and Clinical Aspects of Echinococcosis, a Zoonosis of Increasing Concern, Clin Microbiol Rev. 17 (2004) 107135. doi:10.1128/CMR.17.1.107-135.2004.

[2] D.P. McManus, R.C.A. Thompson, Molecular epidemiology of cystic echinococcosis, Parasitology. 127 Suppl (2003) S37-51.

[3] R.C.A. Thompson, D.P. McManus, Towards a taxonomic revision of the genus Echinococcus, Trends Parasitol. 18 (2002) 452-457.

[4] J. Eckert, R.C. Thompson, Intraspecific variation of Echinococcus granulosus and related species with emphasis on their infectivity to humans, Acta Trop. 64 (1997) 1934.

[5] M. Nakao, A. Lavikainen, T. Yanagida, A. Ito, Phylogenetic systematics of the genus Echinococcus (Cestoda: Taeniidae), Int. J. Parasitol. 43 (2013) 1017-1029. doi:10.1016/j.ijpara.2013.06.002.

[6] M. Nakao, T. Yanagida, S. Konyaev, A. Lavikainen, V.A. Odnokurtsev, V.A. Zaikov, et al., Mitochondrial phylogeny of the genus Echinococcus (Cestoda: Taeniidae) with emphasis on relationships among Echinococcus canadensis genotypes, Parasitology. 140 (2013) 1625-1636. doi:10.1017/S0031182013000565.

[7] M. Nakao, D.P. McManus, P.M. Schantz, P.S. Craig, A. Ito, A molecular phylogeny of the genus Echinococcus inferred from complete mitochondrial genomes, Parasitology. 134 (2007) 713-722. doi:10.1017/S0031182006001934.

[8] J. Knapp, M. Nakao, T. Yanagida, M. Okamoto, U. Saarma, A. Lavikainen, et al., Phylogenetic relationships within Echinococcus and Taenia tapeworms (Cestoda: Taeniidae): an inference from nuclear protein-coding genes, Mol. Phylogenet. Evol. 61 (2011) 628-638. doi:10.1016/j.ympev.2011.07.022.

[9] U. Saarma, I. Jõgisalu, E. Moks, A. Varcasia, A. Lavikainen, A. Oksanen, et al., A novel phylogeny for the genus Echinococcus, based on nuclear data, challenges relationships based on mitochondrial evidence, Parasitology. 136 (2009) 317-328. doi:10.1017/S0031182008005453.

[10] R.C.A. Thompson, A.C. Boxell, B.J. Ralston, C.C. Constantine, R.P. Hobbs, T. Shury, et al., Molecular and morphological characterization of Echinococcus in cervids from North America, Parasitology. 132 (2006) 439-447. doi:10.1017/S0031182005009170.

[11] R.C.A. Thompson, The taxonomy, phylogeny and transmission of Echinococcus, Exp. Parasitol. 119 (2008) 439-446. doi:10.1016/j.exppara.2008.04.016. 
[12] E. Moks, I. Jõgisalu, H. Valdmann, U. Saarma, First report of Echinococcus granulosus G8 in Eurasia and a reappraisal of the phylogenetic relationships of "genotypes" G5G10, Parasitology. 135 (2008) 647-654. doi:10.1017/S0031182008004198.

[13] A.J. Lymbery, E.J. Jenkins, J.M. Schurer, R.C.A. Thompson, Echinococcus canadensis, E. borealis, and E. intermedius. What's in a name?, Trends in Parasitology. 31 (2015) 23-29. doi:10.1016/j.pt.2014.11.003.

[14] M. Nakao, A. Lavikainen, E. Hoberg, Is Echinococcus intermedius a valid species?, Trends Parasitol. 31 (2015) 342-343. doi:10.1016/j.pt.2015.04.012.

[15] A.J. Lymbery, E.J. Jenkins, J.M. Schurer, R.C.A. Thompson, Response to Nakao et al. is Echinococcus intermedius a valid species?, Trends in Parasitology. 31 (2015) 343344. doi:10.1016/j.pt.2015.05.005.

[16] K.L. Haag, A. Zaha, A.M. Araújo, B. Gottstein, Reduced genetic variability within coding and non-coding regions of the Echinococcus multilocularis genome, Parasitology. 115 ( Pt 5) (1997) 521-529.

[17] V. Šnábel, M. Miterpáková, S. D’Amelio, M. Busi, D. Bartková, L. Turčeková, et al., Genetic structuring and differentiation of Echinococcus multilocularis in Slovakia assessed by sequencing and isoenzyme studies, Helminthologia. 43 (2006) 196-202. doi:10.2478/s11687-006-0037-6.

[18] L. Laurimaa, J. Davison, K. Süld, L. Plumer, R. Oja, E. Moks, et al., First report of highly pathogenic Echinococcus granulosus genotype G1 in dogs in a European urban environment, Parasites \& Vectors. 8 (2015) 182. doi:10.1186/s13071-015-0796-3.

[19] T. Romig, D. Ebi, M. Wassermann, Taxonomy and molecular epidemiology of Echinococcus granulosus sensu lato, Vet. Parasitol. (2015). doi:10.1016/j.vetpar.2015.07.035.

[20] J. Knapp, B. Gottstein, U. Saarma, L. Millon, Taxonomy, phylogeny and molecular epidemiology of Echinococcus multilocularis: From fundamental knowledge to health ecology, Vet. Parasitol. 213 (2015) 85-91. doi:10.1016/j.vetpar.2015.07.030.

[21] C.A. Alvarez Rojas, T. Romig, M.W. Lightowlers, Echinococcus granulosus sensu lato genotypes infecting humans - review of current knowledge, Int. J. Parasitol. 44 (2014) 9-18. doi:10.1016/j.ijpara.2013.08.008.

[22] J. Bowles, D. Blair, D.P. McManus, Genetic variants within the genus Echinococcus identified by mitochondrial DNA sequencing, Mol. Biochem. Parasitol. 54 (1992) 165173. 
[23] J. Bowles, D. Blair, D.P. McManus, A molecular phylogeny of the genus Echinococcus, Parasitology. 110 ( Pt 3) (1995) 317-328.

[24] K.M. Gesy, J.M. Schurer, A. Massolo, S. Liccioli, B.T. Elkin, R. Alisauskas, et al., Unexpected diversity of the cestode Echinococcus multilocularis in wildlife in Canada, Int J Parasitol Parasites Wildl. 3 (2014) 81-87. doi:10.1016/j.ijppaw.2014.03.002.

[25] T. Zhang, D. Yang, Z. Zeng, W. Zhao, A. Liu, D. Piao, et al., Genetic characterization of human-derived hydatid cysts of Echinococcus granulosus sensu lato in Heilongjiang Province and the first report of G7 genotype of E. canadensis in humans in China, PLoS ONE. 9 (2014) e109059. doi:10.1371/journal.pone.0109059.

[26] S. Beato, R. Parreira, M. Calado, M.A.A. Grácio, Apparent dominance of the G1-G3 genetic cluster of Echinococcus granulosus strains in the central inland region of Portugal, Parasitol. Int. 59 (2010) 638-642. doi:10.1016/j.parint.2010.08.004.

[27] S. Stefanić, B.S. Shaikenov, P. Deplazes, A. Dinkel, P.R. Torgerson, A. Mathis, Polymerase chain reaction for detection of patent infections of Echinococcus granulosus (“sheep strain") in naturally infected dogs, Parasitol. Res. 92 (2004) 347-351. doi:10.1007/s00436-003-1043-y.

[28] A. Dinkel, M. von Nickisch-Rosenegk, B. Bilger, M. Merli, R. Lucius, T. Romig, Detection of Echinococcus multilocularis in the definitive host: coprodiagnosis by PCR as an alternative to necropsy, J. Clin. Microbiol. 36 (1998) 1871-1876.

[29] G. Boubaker, N. Macchiaroli, L. Prada, M.A. Cucher, M.C. Rosenzvit, I. Ziadinov, et al., A multiplex PCR for the simultaneous detection and genotyping of the Echinococcus granulosus complex, PLoS Negl Trop Dis. 7 (2013) e2017. doi:10.1371/journal.pntd.0002017.

[30] G.B. Santos, S.M. Espínola, H.B. Ferreira, R. Margis, A. Zaha, Rapid detection of Echinococcus species by a high-resolution melting (HRM) approach, Parasit Vectors. 6 (2013) 327. doi:10.1186/1756-3305-6-327.

[31] M. Wassermann, U. Mackenstedt, T. Romig, A loop-mediated isothermal amplification (LAMP) method for the identification of species within the Echinococcus granulosus complex, Vet. Parasitol. 200 (2014) 97-103. doi:10.1016/j.vetpar.2013.12.012.

[32] C.-N. Liu, Z.-Z. Lou, L. Li, H.-B. Yan, D. Blair, M.-T. Lei, et al., Discrimination between E. granulosus sensu stricto, E. multilocularis and E. shiquicus using a multiplex PCR assay, PLoS Negl Trop Dis. 9 (2015) e0004084. doi:10.1371/journal.pntd.0004084.

[33] G. Boubaker, B. Gottstein, A. Hemphill, H. Babba, M. Spiliotis, Echinococcus P29 Antigen: Molecular characterization and implication on post-surgery follow-up of CE 
patients infected with different species of the Echinococcus granulosus complex, PLoS ONE. 9 (2014) e98357. doi:10.1371/journal.pone.0098357.

[34] J.M. Bart, S. Morariu, J. Knapp, M.S. Ilie, M. Pitulescu, A. Anghel, et al., Genetic typing of Echinococcus granulosus in Romania, Parasitol. Res. 98 (2006) 130-137. doi:10.1007/s00436-005-0015-9.

[35] A. Mathis, P. Deplazes, J. Eckert, An improved test system for PCR-based specific detection of Echinococcus multilocularis eggs, J. Helminthol. 70 (1996) 219-222.

[36] D. Trachsel, P. Deplazes, A. Mathis, Identification of taeniid eggs in the faeces from carnivores based on multiplex PCR using targets in mitochondrial DNA, Parasitology. 134 (2007) 911-920. doi:10.1017/S0031182007002235.

[37] N. Saitou, M. Nei, The neighbor-joining method: a new method for reconstructing phylogenetic trees, Mol. Biol. Evol. 4 (1987) 406-425.

[38] M. Kimura, A simple method for estimating evolutionary rates of base substitutions through comparative studies of nucleotide sequences, J. Mol. Evol. 16 (1980) 111-120.

[39] J. Felsenstein, Confidence Limits on Phylogenies: An Approach Using the Bootstrap, Evolution. 39 (1985) 783-791. doi:10.2307/2408678.

[40] M. Nei, S. Kumar, Molecular Evolution and Phylogenetics, Oxford University Press, 2000.

[41] K. Tamura, G. Stecher, D. Peterson, A. Filipski, S. Kumar, MEGA6: Molecular Evolutionary Genetics Analysis Version 6.0, Mol Biol Evol. 30 (2013) 2725-2729. doi:10.1093/molbev/mst197.

[42] W.M. Fitch, Toward Defining the Course of Evolution: Minimum Change for a Specific Tree Topology, Systematic Zoology. 20 (1971) 406-416. doi:10.2307/2412116.

[43] R.V. Eck and M.O. Dayhoff 1966, (n.d.). http://www.megasoftware.net/web_help_7/eck_and_dayhoff_1966.htm (accessed May 17, 2016).

[44] J. Felsenstein, Evolutionary trees from DNA sequences: a maximum likelihood approach, J. Mol. Evol. 17 (1981) 368-376.

[45] J. Felsenstein, Phylogenies and the Comparative Method, The American Naturalist. 125 (1985) 1-15.

[46] B.S. Boufana, M. Campos-Ponce, A. Naidich, I. Buishi, S. Lahmar, E. Zeyhle, et al., Evaluation of three PCR assays for the identification of the sheep strain (genotype 1) of Echinococcus granulosus in canid feces and parasite tissues, Am J Trop Med Hyg. 78 (2008) 777-783. 
[47] I. Abbasi, A. Branzburg, M. Campos-Ponce, S.K. Abdel Hafez, F. Raoul, P.S. Craig, et al., Copro-diagnosis of Echinococcus granulosus infection in dogs by amplification of a newly identified repeated DNA sequence, Am. J. Trop. Med. Hyg. 69 (2003) 324-330.

[48] A. Dinkel, E.M. Njoroge, A. Zimmermann, M. Wälz, E. Zeyhle, I.E. Elmahdi, et al., A PCR system for detection of species and genotypes of the Echinococcus granulosuscomplex, with reference to the epidemiological situation in eastern Africa, Int. J. Parasitol. 34 (2004) 645-653. doi:10.1016/j.ijpara.2003.12.013.

[49] A.K. Rishi, D.P. McManus, Genomic cloning of human Echinococcus granulosus DNA: isolation of recombinant plasmids and their use as genetic markers in strain characterization, Parasitology. 94 ( Pt 2) (1987) 369-383.

[50] B. Boufana, W.S. Lett, S. Lahmar, I. Buishi, A.J. Bodell, A. Varcasia, et al., Echinococcus equinus and Echinococcus granulosus sensu stricto from the United Kingdom: genetic diversity and haplotypic variation, Int. J. Parasitol. 45 (2015) 161166. doi:10.1016/j.ijpara.2014.10.005.

[51] L. Piccoli, C. Bazzocchi, E. Brunetti, P. Mihailescu, C. Bandi, B. Mastalier, et al., Molecular characterization of Echinococcus granulosus in south-eastern Romania: evidence of G1-G3 and G6-G10 complexes in humans, Clin. Microbiol. Infect. 19 (2013) 578-582. doi:10.1111/j.1469-0691.2012.03993.x.

[52] N. Wang, Y. Xie, T. Liu, X. Zhong, J. Wang, D. Hu, et al., The complete mitochondrial genome of G3 genotype of Echinococcus granulosus (Cestoda: Taeniidae), Mitochondrial DNA. (2014) 1-2. doi:10.3109/19401736.2014.961129.

[53] B. Boufana, W. Lett, S. Lahmar, A. Griffiths, D.J. Jenkins, I. Buishi, et al., Canine echinococcosis: genetic diversity of Echinococcus granulosus sensu stricto (s.s.) from definitive hosts, J. Helminthol. 89 (2015) 689-698. doi:10.1017/S0022149X15000395.

[54] M. Isaksson, Å. Hagström, M.T. Armua-Fernandez, H. Wahlström, E.O. Ågren, A. Miller, et al., A semi-automated magnetic capture probe based DNA extraction and realtime PCR method applied in the Swedish surveillance of Echinococcus multilocularis in red fox (Vulpes vulpes) faecal samples, Parasit Vectors. 7 (2014) 583. doi:10.1186/s13071-014-0583-6.

[55] Ø. Øines, M. Isaksson, Å. Hagström, S. Tavornpanich, R.K. Davidson, Laboratory assessment of sensitive molecular tools for detection of low levels of Echinococcus multilocularis-eggs in fox (Vulpes vulpes) faeces, Parasit Vectors. 7 (2014) 246. doi:10.1186/1756-3305-7-246. 


\section{Figure legends:}

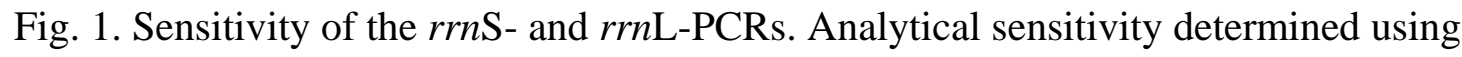
different amounts of E. granulosus s.s. (G1) DNA; Lane M: 100 bp DNA ladder; lanes 1-8, amplicons produced from DNA quantities of $5 \mathrm{ng}, 1 \mathrm{ng}, 100 \mathrm{pg}, 10 \mathrm{pg}, 1 \mathrm{pg}, 100 \mathrm{fg}, 10 \mathrm{fg}$ and $1 \mathrm{fg}$ in a $20 \mu \mathrm{l}$ PCR reaction volume; lane $\mathrm{N}$ : negative control ( $2 \mu 1$ sterile water as a template).

Fig. 2. Specificity of the $r r n L-P C R$

PCR product of the partial $r r n L$ sequence from 7 different cestode species Lane 1: 1 trematode species (Dicrocoelium dendriticum), lane 2-4: 3 nematode species (Ascaris suum, Toxocara canis, and Ancylostoma duodenale), lane 5-11: 7 cestode species (Mesocestoides corti, T. hydatigena, $T$. crassiceps, $T$. polyacantha, $T$. multiceps, $T$. taeniaeformis and Hymenolepis diminuta); Lane M: 100 bp DNA ladder.

Fig. 3. Performance of the rrnS- and rrnL-PCRs using Echinococcus DNAs Agarose gels (1.5\%) displaying PCR products derived from partial mitochondrial $r r n S$ and rrnL gene sequences from Echinococcus reference DNA(s). Lane M: 100 bp DNA ladder; lane 1: E. granulosus s.s. (G1), lane 2: E. granulosus s.s. (G3), lane 3: E. equinus (G4), lane 4: E. ortleppi (G5), lane 5: E. canadensis (G6), lane 6: E. canadensis (G7), lane 7: E. canadensis (G8), lane 8: E. canadensis (G10), lane 9: E. multilocularis, lane 10: E. vogeli and lane $\mathrm{N}$ : negative control ( $2 \mu \mathrm{l}$ sterile water as a template).

Fig. 4. Phylogenetic trees inferred with the Neighbour-Joining (NJ) and based on partial rrnS (A), partial $r r n L(B)$ and concatenated patial gene sequences $r r n L+r r n S$ (C). Neighborjoining (NJ, 1000 replicates), maximum parsimony (MP, 100 replicates) and maximum likelihood (ML, 100 replicates) methods were used to estimate the bootstrap values (shown in the order of NJ/MP/ML). Only bootstrap values higher than $70 \%$ are shown. 
511 Echinococcus reference gDNA samples

\begin{tabular}{|c|c|c|c|c|c|}
\hline $\begin{array}{l}\text { Sample } \\
(g D N A)\end{array}$ & Country & I. host & $\begin{array}{l}\text { Cyst } \\
\text { localization }\end{array}$ & $\begin{array}{l}\text { Echinococcus } \\
\text { spp. }^{\text {a/b }}\end{array}$ & $\begin{array}{l}\text { Echinococcus spp. } \\
\text { (This study) }\end{array}$ \\
\hline Tun_SL321 & Tunisia & sheep & lung & $\begin{array}{l}{ }^{a} \text { E. granulosus s.s } \\
(\mathrm{G} 1 / 2 / 3)\end{array}$ & $\begin{array}{l}\text { E. granulosus s.s. } \\
\text { (G1) }\end{array}$ \\
\hline Tun_SLv346 & Tunisia & sheep & liver & $\begin{array}{l}{ }^{{ }^{a} E \text { E. granulosus s.s }} \\
(\mathrm{G} 1 / 2 / 3)\end{array}$ & $\begin{array}{l}\text { E. granulosus s.s. } \\
\text { (G3) }\end{array}$ \\
\hline Tun_HL315 & Tunisia & $\begin{array}{l}\text { human } \\
\text { origin }\end{array}$ & lung & $\begin{array}{l}{ }^{a} \text { E. granulosus } \\
\text { s.s. }(\mathrm{G} 1 / 2 / 3)\end{array}$ & $\begin{array}{l}\text { E. granulosus s.s. } \\
\text { (G1) }\end{array}$ \\
\hline Alg_SL533 & Algeria & sheep & lung & $\begin{array}{l}{ }^{a} \text { E. granulosus } \\
\text { s.s. }(\mathrm{G} 1 / 2 / 3)\end{array}$ & $\begin{array}{l}\text { E. granulosus s.s. } \\
\text { (G1) }\end{array}$ \\
\hline Alg_SLv312 & Algeria & sheep & liver & $\begin{array}{l}{ }^{a} \text { E. granulosus } \\
\text { s.s. }(\mathrm{G} 1 / 2 / 3)\end{array}$ & $\begin{array}{l}\text { E. granulosus s.s. } \\
\text { (G3) }\end{array}$ \\
\hline Gre_SL276 & Greece & sheep & lung & $\begin{array}{l}{ }^{a} \text { E. granulosus } \\
\text { s.s. }(\mathrm{G} 1 / 2 / 3)\end{array}$ & $\begin{array}{l}\text { E. granulosus s.s. } \\
\text { (G3) }\end{array}$ \\
\hline Ita_S1 & Italy & - & - & $\begin{array}{l}{ }^{a} \text { E. granulosus } \\
\text { s.s. }(\mathrm{G} 1 / 2 / 3)\end{array}$ & $\begin{array}{l}\text { E. granulosus s.s. } \\
\text { (G3) }\end{array}$ \\
\hline Spa_S1 & Spain & donkey & liver & ${ }^{a}$ E. equinus (G4) & E. equinus (G4) \\
\hline Arg_S1 & Argentina & cattle & lung & ${ }^{a}$ E. ortleppi (G5) & E. ortleppi (G5) \\
\hline Alg_Cl12 & Algeria & camel & lung & $\begin{array}{l}{ }^{a} \text { E. canadensis } \\
(\mathrm{G} 6 / 7)\end{array}$ & E. canadensis (G6) \\
\hline Aut_PLv & Austria & pig & liver & $\begin{array}{l}{ }^{a} \text { E. canadensis } \\
\text { (G6/7) }\end{array}$ & E. canadensis $(\mathrm{G} 7)$ \\
\hline Est_CS1 & Estonia & cervid & - & $\begin{array}{l}{ }^{a} \text { E. canadensis } \\
(\mathrm{G} 8 / 10)\end{array}$ & E. canadensis $(\mathrm{G} 8)$ \\
\hline Est_CS2 & Estonia & cervid & - & $\begin{array}{l}{ }^{a} \text { E. canadensis } \\
(\mathrm{G} 8 / 10)\end{array}$ & $\begin{array}{l}\text { E. canadensis } \\
\text { (G10) }\end{array}$ \\
\hline Swi_Em & Switzerland & - & - & ${ }^{b}$ E. multilocularis & E. multilocularis \\
\hline Cnd_Em & Canada & - & - & ${ }^{b}$ E. multilocularis & E. multilocularis \\
\hline Bra_Ev & Brazil & - & - & ${ }^{b}$ E. vogeli & E. vogeli \\
\hline
\end{tabular}

512 a: Echinococcus species was determined by multiplex PCR (Egc-mPCR) performed according to [29].

513 b. Echinococcus species was determined via coxl and nadl sequencing [34]. 
515 Table 2

516 Details of primers used in this study

\begin{tabular}{|l|l|l|l|l|l|}
\hline Gene & Primer & Primer sequence (5' to 3') & $\begin{array}{l}\text { lengt } \\
\text { h }(\mathbf{b p})\end{array}$ & $\begin{array}{l}\text { Amplified } \\
\text { length }(\mathbf{b p})\end{array}$ & $\begin{array}{l}*^{*} \mathbf{T m} \\
\left({ }^{\circ} \mathbf{C}\right)\end{array}$ \\
\hline$r r n S$ & $r r n S-\mathrm{F}$ & GCAAAAGCTGATTAGGG & 17 & 577 & 56 \\
\hline & $r r n S-\mathrm{R}$ & TAACACACAAAAACTC & 16 & & \\
\hline$r r n L$ & $r r n L-\mathrm{F}$ & TTATTTGCCTTTTGCATCA & 19 & 648 & 58 \\
\hline & $r r n L-\mathrm{R}$ & AAAAGATCCTAGGGTCTTTCCGT & 23 & & \\
\hline
\end{tabular}

517

(*) annealing temperature 
Table 3

520 Single nucleotide polymorphisms (SNPs) in partial gene sequences of the $r r n \mathrm{~S}$ and $r r n \mathrm{~L}$

521 genes within E. canadensis (G6/7/8/10)

\begin{tabular}{|l|l|l|l|l|l|l|l|l|l|l|l|l|l|}
\hline rrnS $(\mathbf{5 7 7}$ bp) & 1 & 2 & 3 & 4 & 5 & 6 & 7 & 8 & 9 & 10 & 11 & 12 & \\
\hline Position & $\mathbf{6 6}$ & $\mathbf{9 2}$ & $\mathbf{1 0 2}$ & $\mathbf{1 0 6}$ & $\mathbf{1 0 7}$ & $\mathbf{1 1 0}$ & $\mathbf{1 8 9}$ & $\mathbf{2 2 4}$ & $\mathbf{2 5 5}$ & $\mathbf{4 1 1}$ & $\mathbf{4 2 2}$ & $\mathbf{4 9 5}$ & \\
\hline E. canadensis $(\mathrm{G} 6)$ & A & A & T & G & A & A & G & G & C & A & C & G & \\
\hline E. canadensis $(\mathrm{G} 7)$ & A & A & T & A & A & G & G & G & C & A & C & G & \\
\hline E. canadensis $(\mathrm{G} 8)$ & G & G & T & G & G & T & G & A & C & G & C & A & \\
\hline E. canadensis $(\mathrm{G} 10)$ & G & A & C & G & A & A & A & G & T & A & T & G & \\
\hline rrnL $(\mathbf{6 4 8}$ bp) & I & 2 & 3 & 4 & 5 & 6 & 7 & 8 & 9 & 10 & 11 & 12 & 13 \\
\hline Position & $\mathbf{4 1}$ & $\mathbf{6 2}$ & $\mathbf{7 9}$ & $\mathbf{1 0 9}$ & $\mathbf{1 2 7}$ & $\mathbf{2 0 8}$ & $\mathbf{3 5 0}$ & $\mathbf{3 9 6}$ & $\mathbf{4 4 1}$ & $\mathbf{5 0 9}$ & $\mathbf{5 8 2}$ & $\mathbf{5 9 1}$ & $\mathbf{6 0 3}$ \\
\hline E. canadensis $(\mathrm{G} 6)$ & G & C & A & A & C & G & G & T & A & A & T & A & C \\
\hline E. canadensis $(\mathrm{G} 7)$ & G & C & A & A & C & G & G & T & A & A & T & A & C \\
\hline E. canadensis $(\mathrm{G} 8)$ & G & T & A & A & T & G & A & C & A & G & C & A & T \\
\hline E. canadensis $(\mathrm{G} 10)$ & T & T & G & G & T & A & A & T & C & A & T & G & C \\
\hline
\end{tabular}

